\title{
Multimodal mechanical circulatory assist for perioperative biventricular failure
}

\author{
Yukiharu Sugimura ${ }^{1}$, Arash Mehdiani ${ }^{1}$, Hug Aubin ${ }^{1}$, Philipp Rellecke ${ }^{1}$, Udo Boeken ${ }^{1}$, \\ Artur Lichtenberg ${ }^{2}$, and Payam Akhyari ${ }^{1}$ \\ ${ }^{1}$ Heinrich-Heine-Universitat Dusseldorf \\ ${ }^{2}$ University Hospital Dusseldorf
}

September 11, 2020

\begin{abstract}
A 61-year-old woman with acute myocardial infarction (MI), cardiogenic shock and Impella CP support underwent emergency coronary artery bypass grafting. Postoperatively venous-arterial extracorporeal membrane oxygenation (va-ECMO) became necessary, followed by Impella 5.0 insertion on 7th postoperative day (POD), addition of right ventricular support by TandemHeart due to inadequate flow of Impella system, which then allowed for va-ECMO weaning. Impella und TandemHeart were removed on 14th POD, 31st POD, respectively. Biventricular decompensation following MI was successfully treated by a sequence of different mechanical circulatory support systems allowing an adaptive weaning strategy.
\end{abstract}

\section{Introduction:}

Cardiogenic shock (CS) after acute myocardial infarction (AMI) or sustained heart failure after coronary artery bypass grafting (CABG) for AMI may be refractory to conservative treatment and require mechanical circulatory support. We report a case of successful sequential recovery with multimodal mechanical support systems in sustained CS pre and post CABG.

\section{Case Report:}

A 61-year-old woman presenting with dyspnea and acute chest pain on an emergency base rapidly was diagnosed with anterolateral ST-elevation myocardial infarction (Creatinin kinase (CK); 2498 U/l, CK-MB; $173 \mathrm{U} / \mathrm{l}$, Troponin T; $5668 \mathrm{ng} / \mathrm{l})$. Coronary angiography revealed long segment severe stenosis of left anterior descending (LAD), severe stenosis in the proximal marginal branch of circumflex artery (RM), and segment 2 of the right coronary artery (Fig 1). Due to CS, Impella CP (by Abiomed) was implanted in the cath lab through the right femoral artery as "bridge therapy," and the patient was immediately transferred to undergo CABG. Salvage on-pump CABG was performed with coronary bypasses to the LAD by the left mammary artery, to RM and posterior descending artery by a saphenous vein segment. In order to avoid damage to the Impella during aortic cross clamping and proximal anastomosis of the bypass grafts Impella CP was removed. Due to severe LV dysfunction venous-arterial extracorporeal membrane oxygenation (va-ECMO; Centrimag, by Abbott) via the right femoral vessels was inserted. Myocardial markers showed a satisfactory course 6 hours after CABG with clear drop in CK (1504 U/l) and CK-MB (58U/1).

On $7^{\text {th }}$ postoperative day (POD), however, transthoracic echocardiography (TTE) showed still severely impaired systolic LV function with global hypokinesia and moderate distention (ejection fraction; EF 20\%), while respiratory function showed no signs of impairment. Therefore, it was decided to attempt a switch from va-ECMO to isolated temporary left ventricular support and unloading by Impella 5.0 in the intention of downgrading the level of support. In the operation room, a $10 \mathrm{~mm}$ vascular graft was anastomosed to the 
right axillary artery. However, under transesophageal echocardiography (TEE) as well as fluoroscopic control the Impella 5.0 could not be introduced beyond the subclavian artery. In this situation, the strategy was changed, and the arterial line of the va-ECMO was first transferred from the femoral artery to the axillary artery via already existing prosthesis. Then a further $10 \mathrm{~mm}$ silver-coated prostheses was sewn on the right common femoral artery, and Impella 5.0 was successfully implanted.

Nevertheless, the weaning of va-ECMO was impossible with maximum flow rates through Impella 5.0 ranging 2.0-2.5 L/min under P3-4 and additional va-ECMO support of ca. $2.5 \mathrm{~L} / \mathrm{min}$ (Fig. 2a). The patient was transferred to the ICU and demonstrated signs of right ventricle (RV) failure with central venous pressure $23 \mathrm{mmHg}$ when va-ECMO weaning below $1.5 \mathrm{l} / \mathrm{min}$ was attempted. Therefore, a percutaneous RV assisted device (Tandem-Heart ProtekDuo by LivaNova; run by Centrimag pump) with integrated oxygenator was implanted via the right internal jugular vein on $10^{\text {th }} \mathrm{POD}$, allowing termination of va-ECMO therapy and enabling Impella 5.0 flows reaching a full range of $5.0 \mathrm{~L} / \mathrm{min}$ for the first time (Fig. 2b) .

On $14^{\text {th }}$ POD, TEE demonstrated an external compression of RA and RV. After hematoma removal via re-sternotomy TEE showed sufficient recovery of LV and Impella could be removed. The oxygenator was withdrawn from the RVAD support on $27^{\text {th }}$ POD. TTE demonstrated LVEF recovered to $50 \%$ with hypokinesis at the septal and posterior wall, mild mitral regurgitation, mild to moderately depressed RV function. After pharmacological conditioning with Levosimendan, Tandem-Heart was successfully removed on $31^{\text {st }}$ POD. Due to wound healing disorders a further treatment by plastic and reconstructive surgery became necessary and the patient was finally discharged for rehabilitation on the $160^{\text {th }}$ postoperative day.

\section{Discussion:}

After disappointing results of intra-aortic balloon pumping ${ }^{1-3}$, va-ECMO has been the preferred option of the mechanical circulatory support for sustained CS. However, va-ECMO does not unload LV, and by increasing the afterload, it may lead to LV congestion, pulmonary edema, and secondary right heart failure.

Recently, Impella (2.5, CP, and 5.0) has evolved as another valid catheter-guided mechanical circulatory device for left heart failure. Impella can produce antegrade flow and additionally unload LV, i.e., reduce myocardial oxygen demand and increase the coronary circulation ${ }^{4-7}$.

Back to this case, a sequential combination of different systems allowed for successful step-wise recovery of a patient initially at a very marginal state with sustained biventricular failure. Impella $\mathrm{CP}$ saved the patient in primary low-output and served as a bridge-to-revascularization. Termination of the heart-lung machine was only possible after implantation of va-ECMO. With no sign of relevant LV recovery on $7^{\text {th }}$ POD, Impella 5.0 was implanted to terminate va-ECMO, and the Impella was inserted through the femoral artery as the axillar access was impractical. Retrospectively, we speculate that 1) either the prosthesis was sewn in a region not allowing flexible manipulation and introduction of the big caliber of Impella 5.0 (the pump size is max. $21 \mathrm{Fr}$; or 2) due to a vertical angle between the prosthesis and the artery (ideally 45 to 60 degree); or 3) due to additional borderline diameter of the axillary artery. In a retrospective view, both the inadequate flow of Impella 5.0 and the impossibility of va-ECMO weaning were most likely consequences of RV dysfunction. Actually, Impella 5.0 delivered $5.0 \mathrm{~L} / \mathrm{min}$ flow after implantation of TandemHeart ProtekDuo. TandemHeart ProtekDuo is a percutaneous device for RV support and a valid alternative to surgically implanted RV assisted device, avoiding additional operative steps. In our opinion, a stepwise strategy and adaptive weaning pattern with switch from va-ECMO to Impella 5.0 for the LV support with implantation of TandemHeart ProtekDuo for the RV support and consecutive weaning of either univentricular support under differentiated pharmacological conditioning and close TEE monitoring is a favorable approach for successful stabilization of critically unstable patients in CS such as in the present case.

\section{Conclusion:}

Biventricular decompensation following MI was successfully treated by a sequence of different mechanical circulatory support systems allowing an adaptive weaning strategy.

Ethical approval: 
This manuscript followed the principles of the Declaration of Helsinki and the Declaration of Istanbul. The appropriate informed consent was obtained for the publication of this manuscript.

\section{References:}

1. Thiele H, Zeymer U, Thelemann N, et al. Intraaortic Balloon Pump in Cardiogenic Shock Complicating Acute Myocardial Infarction: Long-Term 6-Year Outcome of the Randomized IABP-SHOCK II Trial.Circulation. 2018.

2. Thiele H, Zeymer U, Neumann FJ, et al. Intra-aortic balloon counterpulsation in acute myocardial infarction complicated by cardiogenic shock (IABP-SHOCK II): final 12 month results of a randomised, open-label trial. Lancet. 2013;382(9905):1638-1645.

3. Thiele H, Zeymer U, Neumann FJ, et al. Intraaortic balloon support for myocardial infarction with cardiogenic shock. N Engl J Med.2012;367(14):1287-1296.

4. Griffith BP, Anderson MB, Samuels LE, Pae WE, Jr., Naka Y, Frazier OH. The RECOVER I: a multicenter prospective study of Impella 5.0/LD for postcardiotomy circulatory support. J Thorac Cardiovasc Surg.2013;145(2):548-554.

5. Aqel RA, Hage FG, Iskandrian AE. Improvement of myocardial perfusion with a percutaneously inserted left ventricular assist device. J Nucl Cardiol. 2010;17(1):158-160.

6. Remmelink M, Sjauw KD, Henriques JP, et al. Effects of left ventricular unloading by Impella recover LP2.5 on coronary hemodynamics. Catheter Cardiovasc Interv. 2007;70(4):532-537.

7. Meyns B, Stolinski J, Leunens V, Verbeken E, Flameng W. Left ventricular support by catheter-mounted axial flow pump reduces infarct size. J Am Coll Cardiol. 2003;41(7):1087-1095.

Figure legends:

Fig. 1. Preoperative coronary angiography revealed (a) long segment multiple severe stenoses of left anterior descending, (b) severe stenosis in the proximal first marginal branch and downstream second stenosis of circumflex artery, and (c) segment 2 of the right coronary artery and posterior descending artery. (Black arrows show relevant stenosis)

Fig. 2. Postoperative chest radiography. (a). Initial support with venous-arterial extracorporeal membrane oxygenation (black line and arrows: venous cannula, white line and arrows: Impella; 7th postoperative day (POD)); (b) later downgrading of support to Impella (white line and arrows) combined with TandemHeart catheter (black dotted line and arrows; 11th POD). 

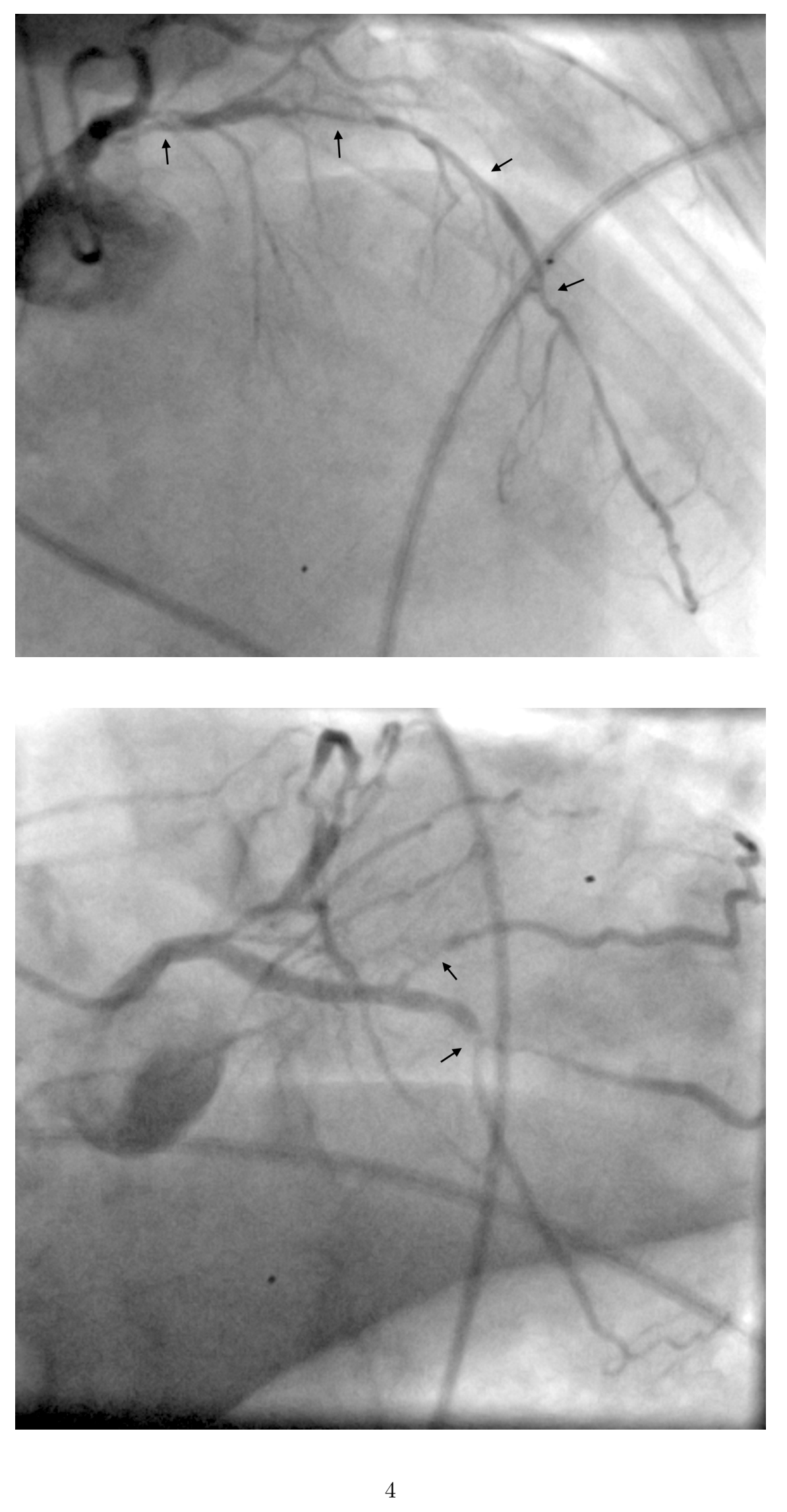

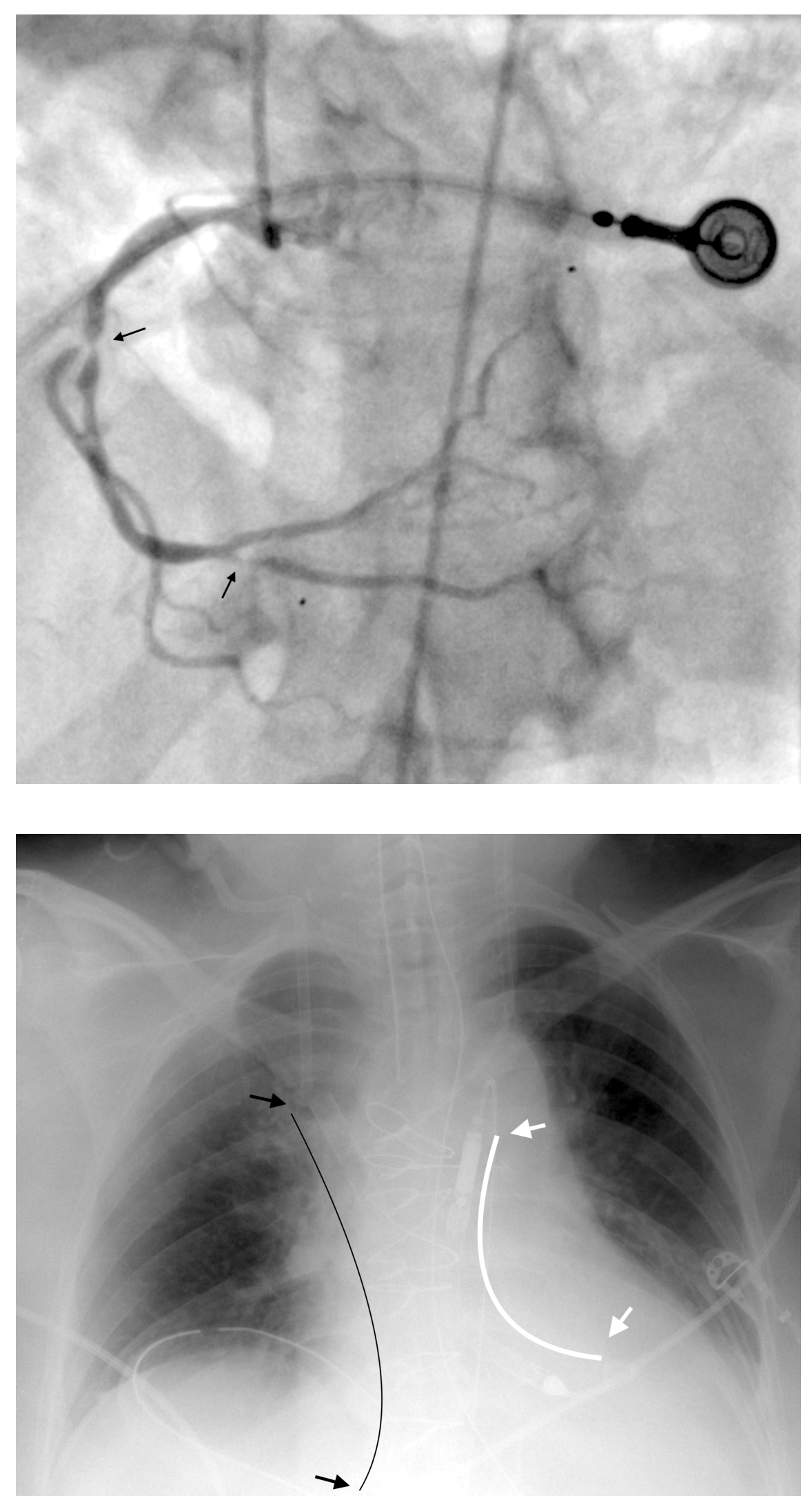


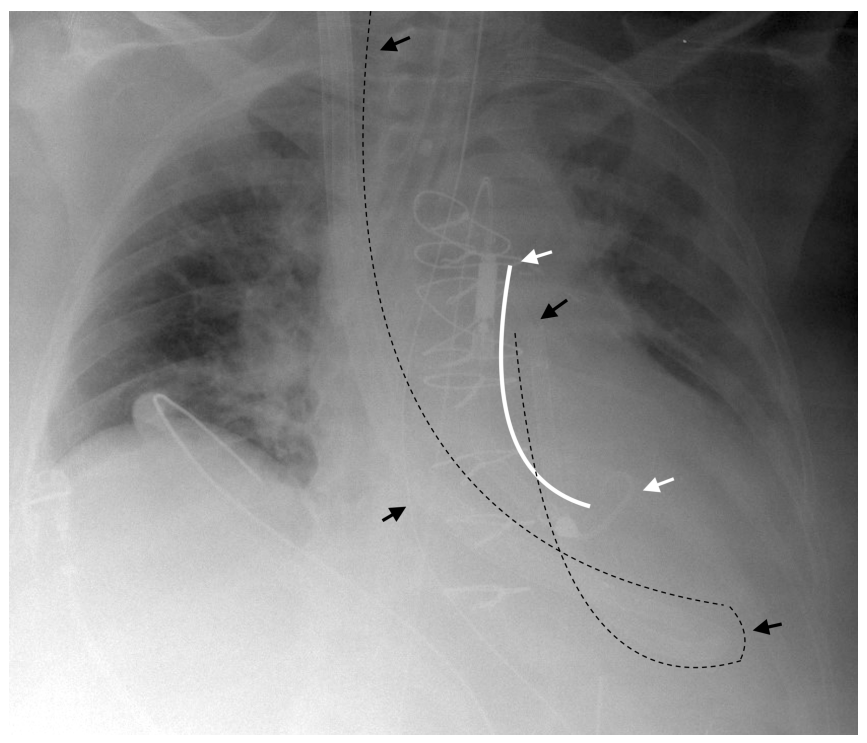

